\title{
Engineering hydrogel viscoelasticity
}

Ludovica Cacopardo $^{1,2}$, Nicole Guazzelli ${ }^{1}$, Roberta Nossa ${ }^{1,2}$, Giorgio Mattei ${ }^{2,3,4}$ and Arti Ahluwalia ${ }^{1,2, *}$

${ }^{I}$ Research Centre "E. Piaggio", University of Pisa, Largo Lucio Lazzarino 1, 56122 Pisa, Italy

${ }^{2}$ Department of Information Engineering, University of Pisa, Via Girolamo Caruso 16, 56122 Pisa, Italy

${ }^{3}$ Optics11 B.V., De Boelelaan 1081, 1081 HV Amsterdam, The Netherlands

${ }^{4}$ Biophotonics \& Medical Imaging and LaserLaB, VU University Amsterdam, De Boelelaan 1105, $1081 \mathrm{HV}$ Amsterdam, The Netherlands

${ }^{*}$ Corresponding Author

Address: Research Centre "E. Piaggio", University of Pisa, Largo Lucio Lazzarino 1, 56122 Pisa, Italy

Tel: +39050 2217056

Fax: +390502217051

E-mail: arti.ahluwalia@ unipi.it 


\begin{abstract}
The aim of this study was to identify a method for modifying the time-dependent viscoelastic properties of gels without altering the elastic component. To this end, two hydrogels commonly used in biomedical applications, agarose and acrylamide, were prepared in aqueous solutions of dextran with increasing concentrations $(0,2$ and $5 \% \mathrm{w} / \mathrm{v})$ and hence increasing viscosities. Commercial polyurethane sponges soaked in the same solutions were used as controls, since, unlike in hydrogels, the liquid in these sponge systems is poorly bound to the polymer network. Sample viscoelastic properties were characterised using the epsilon-dot method, based on compression tests at different constant strain-rates. Experimental data were fitted to a standard linear solid model. While increasing the liquid viscosity in the controls resulted in a significant increase of the characteristic relaxation time $(\tau)$, both the instantaneous $\left(E_{\text {inst }}\right)$ and the equilibrium $\left(E_{e q}\right)$ elastic moduli remained almost constant.
\end{abstract}

However, in the hydrogels a significant reduction of both $E_{\text {inst }}$ and $\tau$ was observed. On the other hand, as expected, $E_{e q}-$ an indicator of the equilibrium elastic behaviour after the occurrence of viscoelastic relaxation dynamics - was found to be independent of the liquid phase viscosity.

Therefore, although the elastic and viscous components of hydrogels cannot be completely decoupled due to the interaction of the liquid and solid phases, we show that their viscoelastic behaviour can be modulated by varying the viscosity of the aqueous phase. This simple-yet-effective strategy could be useful in the field of mechanobiology, particularly for studying cell response to substrate viscoelasticity while keeping the elastic cue (i.e. equilibrium modulus, or quasi-static stiffness) constant.

Keywords: viscoelasticity, damping component modulation, agarose, polyacrylamide, mechanobiology

\title{
1. Introduction
}

Hydrogels are characterised by a biphasic structure: a solid network, which confers elastic properties, surrounded by an aqueous solution, which is associated with their viscous behaviour (Hoffman, 2012). Since hydrogel composition and mechanical properties are similar to those of biological tissues, they are of great interest as biomaterials. Numerous reports on engineering hydrogels to better recapitulate the in-vivo milieu focus on characterising and tuning their elastic behaviour, typically through the use of crosslinking strategies (e.g. physical, chemical, enzymatic) (Hoffman, 2012) and/or adding reinforcements (e.g. fibres, fillers) (Bhattacharya et al., 2006). This is likely due to the wealth of literature on cell response to stiffness (Humphrey et al., 2014; Ingber, 1997; Wells, 2008). In particular, polyacrylamide (PAAm) is the workhorse of mechanobiology studies and has been used extensively (Awad et al., 2004; Adam J. Engler et al., 2006; Jay et al., 2009; Kumar, 2014; Nemir and West, 2009; Tse and Engler, 2010; Yang et al., 2011). In a seminal work, Engler and co-workers demonstrated that stem cells alter their commitment depending on substrate stiffness using PAAm hydrogels prepared with different concentrations of bis-acrylamide (Adam J Engler et al., 2006). A few years later, Pek et al. reported on the differentiation of 3D mesenchymal stem cell (MSC) cultures in thixotropic polyethylene glycol silica gels (Pek et al., 2010). In particular, they observed different levels of neural, myogenic and osteogenic factor expression as the gel liquefaction stress (i.e. minimum stress required to liquefy the hydrogel) increases. Moreover, the expression of these factors was higher in the 3D gels in comparison to the $2 \mathrm{D}$ culture, indicating that the modulation of stiffness of a three-dimensional environment is more effective on cell differentiation. Stiffness is also fundamental for cellular migration within 3D matrices. Zaman et al. showed that keeping biochemical parameters (such as matrix ligands) constant, the migration speed of human prostate carcinoma cells was higher within soft scaffolds (Zaman et al., 2006). Finally, neurite extension in agarose gels with different concentration was found to be inversely proportional to the stiffness of the gels (Balgude et al., 2001).

However, hydrogels as well as biological tissues are intrinsically viscoelastic. Recognising this, some attention has recently been directed to tuning material viscoelasticity thanks to studies demonstrating that cells also respond to the time-dependent properties of their substrates. PAAm gels with different viscous properties can be realized by adding high molecular weight linear polymers, which are sterically entrapped in the gels (Charrier et al., 2018), or simply by varying the proportion of acrylamide monomer and bis-acrylamide (Cameron et al., 2011). Using the former method, Charrier and colleagues generated gels with a loss modulus $\left(\mathrm{G}^{\prime \prime}\right)$ of 200 and $500 \mathrm{~Pa}$ for $1.8 \%$ and $2.75 \% \mathrm{w} / \mathrm{v}$ of polymer. The authors reported that fibroblast spreading 
decreases with increasing gel viscosity and that this effect is different in case of collagen or fibronectin coatings, suggesting that different biochemical pathways influence cell sensitivity to viscosity. Moreover, they showed that for a constant stiffness (storage modulus $\mathrm{G}^{\prime}$ ), the differentiation of primary rat hepatic stellate cells to fibrogenic myofibroblasts on stiff substrates decreases as the viscosity increases. The authors hypothesize that cell traction forces decrease in response to relaxation phenomena (Charrier et al., 2018). Cameron and coworkers prepared PAAm hydrogels with different ratios of acrylamide and bis-acrylamide. The values of $\mathrm{G}^{\prime \prime}$ at $0.005 \mathrm{rad} \cdot \mathrm{s}^{-1}$ were 130,10 and $1 \mathrm{~Pa}$ for $15 / 0.0125 \%, 12 / 0.0358 \%$ and 8/0.1\% w/v acrylamide/crosslinker weight ratio, respectively. They observed increased spreading area and proliferation of MSCs and a higher tendency to differentiate into different lineages as the viscosity increased. Thus, they conclude that "a reduction in both passive and actively generated isometric cytoskeletal tension is caused by the inherent creep of substrates with a high loss modulus" (Cameron et al., 2011). Finally, thanks to a computational model and cell experiments on alginate hydrogels, Chaudhuri and coworkers showed that cell spreading is higher on ionically crosslinked viscoelastic soft gels with respect to covalently cross-linked stiff elastic ones (Chaudhuri et al., 2015).

From this short overview, it is clear that to date hydrogel viscoelastic properties have been modulated by acting on the solid network, e.g. by varying the polymer and/or crosslinker concentration. This is likely to alter not only the hydrogel viscoelastic dynamics, described by one (or more) characteristic relaxation time(s), but also the residual equilibrium elastic properties after the occurrence of viscoelastic phenomena, described by the equilibrium elastic modulus. Indeed, we recently reported that glutaraldehyde-crosslinked gelatin hydrogels showed increased instantaneous and equilibrium elastic moduli as well as characteristic relaxation time with increasing crosslinker concentration, meaning that - for the same polymer concentration - more crosslinked hydrogels are not only stiffer but there is also a concomitant change in their viscoelastic behaviour towards a more elastic one (Mattei et al., 2017).

Given the emerging importance of hydrogel viscoelastic properties in regulating cell behavior and lineage commitment, it could be of interest to decouple viscoelastic characteristics from elastic ones, by modulating viscoelastic relaxation dynamics while keeping the equilibrium elastic modulus constant. To achieve this, we chose to act on the hydrogel liquid phase rather than the solid polymer network. In particular, we investigated the effect of changing the viscosity of the aqueous phase in agarose and polyacrylamide (PAAm) hydrogels through the addition of dextran. Dextran is a homo-polysaccharide of glucose containing consecutive $\alpha(1,6)$ linkages, which contribute to the high solubility of the molecule in water (Naessens et al., 2005). The molecule is often used to increase the viscosity of aqueous solutions, for example, to mimic blood viscosity (Campolo et al., 2014; Liepsch et al., 1991). Agarose is a linear polysaccharide that is normally insoluble in organic solvents, but highly soluble in water. Its gelling capacity is strongly dependent on the interaction with water and temperature. Its compressive stiffness varies as a function of its concentration and physical gels, stable at an ambient temperature of around $20-25^{\circ} \mathrm{C}$, are formed without external crosslinkers. Water molecules bonded to this system contribute to the stability of the agarose double helix and to the stiffness of the gel (Arnott et al., 1974; Nijenhuis, 1997). On the other hand, as reviewed above, the stiffness of PAAm depends not only on the initial quantity of monomer used, but also on the quantity of crosslinker and initiators (Keplinger et al., 2013). Porous polyurethane (PU) sponges soaked in dextran solutions were used as controls. Since the liquid phase is not bound to the polymer network in these sponges, they are not biphasic gel systems. Thus, unlike agarose and PAAm hydrogels, the interaction between the solid sponge and liquid filling its pores is weak, therefore their viscoelastic properties should be directly related to the viscosity of the liquid phase. The compressive viscoelastic properties of both PU sponges and agarose and PAAm hydrogels were measured at various constant strain rates, using the epsilon dot method (Tirella et al., 2013). Stress-time data collected at different strain rates were then globally fitted to a Maxwell standard linear solid (SLS) model to determine the effect of increasing dextran solution viscosity on the viscoelastic parameters, i.e. the instantaneous and equilibrium elastic moduli, and the characteristic relaxation time.

\section{Material and Methods}

\subsection{Sample Preparation}


Agarose solutions were prepared at $1 \% \mathrm{w} / \mathrm{v}$ polymer concentration by dissolving agarose powder (Sigma A9539) in aqueous media with different viscosities using 0 and 2 and 5\% w/v dextran (Sigma D1037, MW 425-570000). In the range of dextran molecular weights and at the concentrations used, the aqueous phases are Newtonian (Guo et al., 2013). The solutions were stirred until boiling and then cast into custom moulds, obtaining $13 \mathrm{~mm}$ diameter $-8 \mathrm{~mm}$ height cylindrical samples.

PAAm gels were prepared by dissolving $15.6 \% \mathrm{w} / \mathrm{v}$ of Acrylamide monomer (Biorad) in 0,2 and $5 \%$ dextran in water with the addition of $0.02 \% \mathrm{w} / \mathrm{v}$ Bis-acrylamide (N,N0-methylene bis-acrylamide, Sigma M7256), $0.084 \% \mathrm{w} / \mathrm{v}$ of ammonium persulfate (Biorad) and $0.056 \% \mathrm{v} / \mathrm{v}$ Tetramethylethylenediamine (TEMED) (Sigma T9281). All the components were mixed and the final solution was first degassed for 2 minutes and then cast in the custom molds. The polymerization was completed in 90 minutes at room temperature.

Commercial PU sponges (UR303810, water adsorption 0.1\%, Goodfellow, Huntingdon - UK) were cut into cubes (sides $\simeq 10 \mathrm{~mm}$ ) to obtain control samples which were submerged in the different aqueous media for about 30 minutes at room temperature to allow fluid absorption. They were also tested in dry conditions to obtain a baseline viscoelastic behaviour in the absence of liquids.

\subsection{Viscosity Measurements}

The dynamic viscosity $(\mu)$ of the different aqueous phases $(0 \%, 2 \%$ and $5 \%$ dextran) was characterised with an AMVN Automated micro-viscometer (Anton Paar GmbH, Germany), which measures the rolling time of a ball through transparent and opaque liquids according to Höppler falling ball principle (Kulicke and Clasen, 2013). For each solution, both density and viscosity are measured within the range of room temperature $\left(16^{\circ} \mathrm{C}\right.$, $21^{\circ} \mathrm{C}$ and $26^{\circ} \mathrm{C}$ ) using a capillary with $1.6 \mathrm{~mm}$ inner diameter and a $1.5 \mathrm{~mm}$ diameter ball. Six repeats were performed for each measurement.

\subsection{Mechanical Testing and Statistical Analysis}

The viscoelastic properties of the sponges and gels were tested using the epsilon-dot method (Tirella et al., 2013). Unconfined bulk compression tests were performed at different constant strain rates $(0.0005,0.001$, 0.005 and $0.01 \mathrm{~s}^{-1}$ ) with a Zwick/Roell ProLine Z005 uniaxial testing device (Germany). All samples except the dry PU sponges were tested while being partially submerged in their respective aqueous phase to maintain hydration. Measurements at each strain rate were performed on $n=3$ different samples to avoid any effect due to repeated testing cycles, leading to a total of 4 (strain rates) $\times 3$ (replicates) $=12$ samples tested per material investigated. For each sample engineering stress-time curves recorded at the same strain-rate were averaged. Then, the mean curves obtained at the different strain rates investigated were globally fitted to a standard linear solid (SLS) model as described in (Tirella et al., 2013), obtaining $E_{1}, E_{2}$ and $\eta$ (Figure 1), which were used to compute the instantaneous $\left(\mathrm{E}_{\mathrm{inst}}=\mathrm{E}_{1}+\mathrm{E}_{2}\right)$ and equilibrium $\left(\mathrm{E}_{\mathrm{eq}}=\mathrm{E}_{1}\right)$ elastic moduli and the characteristic relaxation time $\left(\tau=\eta / E_{2}\right)$. In particular, $E_{\text {inst }}$ refers to the initial elastic response, which is given by the sum of all the springs in the lumped model since the viscous components are "shorted" out and do not deform while sustaining the load. The equilibrium modulus refers instead to the "static" response after the viscoelastic dynamics have occurred, and it is given by $\mathrm{E}_{1}$, as the viscous components are on "open circuit", so they cannot sustain any load or stress. Finally, a 1-way ANOVA analysis was performed to investigate the effect of varying the dextran solution concentration on the resultant viscoelastic properties.

\section{Results}

The dynamic viscosity, $\mu$, of the solutions as a function of dextran concentration and temperature $(16,21$ and $26^{\circ} \mathrm{C}$ ) is shown in Figure 2. As expected, $\mu$ increases with increasing dextran concentration and decreases with temperature.

The results of the mechanical tests for the sponges are reported in Figure 3: the relaxation time increases significantly with increasing dextran concentration; conversely, both $\mathrm{E}_{\text {inst }}$ and $\mathrm{E}_{\mathrm{eq}}$ are fairly constant for the wet sponges. This indicates that only the SLS dashpot parameter changes, while both SLS springs remain unaltered as the viscosity increases. The dry sponges have significantly higher $\mathrm{E}_{\text {inst }}(74.47 \pm 4.22 \mathrm{kPa})$ and $\mathrm{E}_{\text {eq }}$ $(25.39 \pm 0.49 \mathrm{kPa})$ as well as a significantly lower $\tau(1.60 \pm 0.21 \mathrm{~s})$ than the wet ones. 
Figure 4 illustrates the results obtained for agarose gels: the increase in viscosity of the liquid phase resulted in a significant reduction of both instantaneous elastic modulus and relaxation time, indicating that the viscoelastic components of the system (i.e. $\mathrm{E}_{2}$ and $\eta$, which are placed in series in the SLS parallel model, Figure 1) are altered. The $E_{\text {eq }}$ (equivalent to $E_{1}$ in Figure 1) does not vary significantly between agarose gels prepared in 0 and $2 \% \mathrm{w} / \mathrm{v}$ dextran solutions, while it was significantly reduced for hydrogels prepared in $5 \%$ w/v dextran.

In the case of PAAm gels (Figure 5), $\mathrm{E}_{\mathrm{inst}}$ and $\tau$ follow the same decreasing trend observed for agarose, while the equilibrium elastic modulus is almost constant with increasing dextran concentration. The ANOVA analysis shows a significant reduction of both instantaneous elastic modulus and characteristic relaxation time with increasing dextran concentration.

\section{Discussion}

In the control PU sponges, there is little or no interaction between the spongiosum structure and the liquid phase. Water diffuses inside the small pores formed during the manufacture of foams, which are produced by adding inert gases to the monomers precursors to induce bubble formation (Sabbahi and Vergnaud, 1993). Thus, the only factor which conditions the movement of water through the pores during unconfined compression is the viscosity of the aqueous solution. This means that water (which has a lower viscosity than dextran) needs less time to re-distribute through the pores, resulting in a lower relaxation time $\tau$. Increasing dextran concentration and hence $\mu$, results in a higher $\tau$ because the fluid resists motion.

The water swollen hydrogels networks cannot be considered in the same light as the PU. In particular, the aqueous phase is formed of both 'bound water' and 'free water'. The latter is defined as the amount of water that does not interact with the polymeric chains as all the hydrophilic and hydrophobic groups have been saturated with the 'primary and secondary bound water' respectively. As reported by Hoffman et al. the 'free water' is assumed to fill the space between network chains (Hoffman, 2012), thus it is able to redistribute within the network when the gel is deformed.

Increasing the dextran concentration in the liquid phase interferes with hydrogen bonding between agarose chains and water, allowing the latter to move more easily through the hydrogel molecular pores and redistribute within its network (Nijenhuis, 1997). This results in a reduction in compressive stiffness as well as a decrease in relaxation time because water moves faster as it is less bound to the network.

PAAm gels are highly hydrophilic but present a low hydrolytic stability (Dursun et al., 2004). Their gelification occurs through the chemical crosslinking between acrylamide and bis-acrylamide (N,N0-methylene bisacrylamide, in our case), which results in the formation of a porous network with covalent bonds between polymer chains (Dursun et al., 2004). In the samples used here, despite the fact that the instantaneous elastic moduli of the agarose and PAAm gels in the absence of dextran were fairly similar, the latter gels have a lower $\tau$ and exhibit a higher difference between instantaneous and equilibrium elastic modulus than the former. It is likely therefore that, even in the absence of dextran, water molecules are less bound to the PAAm network than they are to agarose, and this effect is amplified with increasing amounts of the polysaccharide. As in the case of agarose, our results suggest that as dextran concentration increases water moves more easily through the network resulting in a lower relaxation time. Our results are supported by the findings of Wu and Shanks who studied how PAAm solutions behave with different glucose concentrations. In particular, they found that when hydrogen bonds between glucose and water overwhelm the bonds between PAAm amides and water, the acrylamide intramolecular interactions become higher than intermolecular ones with the result that the amount of 'free water' increases. i.e. water is less bound to the chains and thus moves more easily through the polymeric network (Wu and Shanks, 2003). This concept, which is schematised in Figure 6, can also be extended to dextran, as it is a polysaccharide composed of different glucose molecules (Hammond, 1969). The figure illustrates the formation of hydrogen bonds between the solid network and water in the absence of dextran and the tendency of water to preferentially form bonds with the polysaccharide rather than with PAAm when it is present in the hydrogel liquid phase.

In unconfined compression experiments $\mathrm{E}_{\mathrm{eq}}$ in principle represents the elastic modulus of the polymer network at equilibrium, i.e. after the viscoelastic dynamics have occurred and thus when the viscous component cannot support any load. We hypothesise that $\mathrm{E}_{\mathrm{eq}}$ does not vary significantly in the hydrogels because the addition of 
dextran does not modify the elastic network itself, but only the interaction between the liquid and solid phase. On the other hand, $\mathrm{E}_{\text {inst }}$ changes according to the degree of water-polymer binding. Specifically, the instantaneous elastic modulus is higher for a network strongly bound to water and lower in the case of weak water-polymer interactions. This suggests that varying the viscosity of the liquid phase in hydrogels by adding components to the aqueous solution may alter the interaction between the liquid phase and the polymeric network and thereby modify the bulk viscoelastic response. In our case, dextran reduced this interaction in both agarose and PAAm gels, facilitating liquid phase movement (and hence decreasing the relaxation time). On the contrary, when these interactions are lower or absent, as in the case of the sponges, increasing the viscosity of the liquid phase results in an increase of $\tau$, since the time required for the liquid phase to move is higher. Notably, the mechanical properties derived in the strain-rate domain (as presented in this work) have been demonstrated to be equivalent to those obtained via frequency domain testing (e.g. dynamic mechanical analysis, DMA). In particular, frequency-domain storage and loss moduli can be estimated from strain-rate measurements, and - dually - strain rate domain viscoelastic constants $\left(\mathrm{E}_{\mathrm{inst}}, \mathrm{E}_{\mathrm{eq}}\right.$, and $\left.\tau_{\mathrm{s}}\right)$ can be obtained from frequency tests (Bartolini et al., 2018; Zeltmann et al., 2016).

\section{Conclusions}

To date, most mechanobiology studies have focused on characterizing biological tissue stiffness and investigating cell behavior on substrates, typically hydrogels, on the bases of their elasticity. However, both hydrogels and biological tissues are intrinsically viscoelastic. In light of this, methods to modulate hydrogels viscoelastic properties have been recently proposed to study cell response to substrate viscoelasticity. In conclusion, as an alternative to the crosslinking of the polymer network, we demonstrate that modifying the viscosity of the hydrogel liquid phase can also modulate its viscoelastic properties. Notably, this strategy allows changing the mechanical properties reflecting viscoelastic phenomena (i.e. $E_{2}$ and $\eta$, see Figure 1 ) while keeping the equilibrium elastic response $\left(\mathrm{E}_{\mathrm{eq}}=\mathrm{E}_{1}\right)$ constant. This decoupling is generally not achievable when using strategies that target the hydrogel polymer network, as they usually alter both the hydrogel viscoelastic dynamics and the equilibrium elastic response. Future studies will be focused on investigating the contribution of the liquid phase in different hydrogels with particular regard to the interaction between the polymer network and the liquid phase, given its critical role in determining the resultant bulk viscoelastic behaviour.

\section{References}

Arnott, S., Fulmer, A., Scott, W.E., Dea, I.C.M., Moorhouse, R., Rees, D.A., 1974. The agarose double helix and its function in agarose gel structure. J. Mol. Biol. 90, 269-284. https://doi.org/10.1016/00222836(74)90372-6

Awad, H.A., Wickham, M.Q., Leddy, H.A., Gimble, J.M., Guilak, F., 2004. Chondrogenic differentiation of adipose-derived adult stem cells in agarose, alginate, and gelatin scaffolds. Biomaterials 25, 32113222. https://doi.org/10.1016/j.biomaterials.2003.10.045

Balgude, A.P., Yu, X., Szymanski, A., Bellamkonda, R. V., 2001. Agarose gel stiffness determines rate of DRG neurite extension in 3D cultures. Biomaterials 22, 1077-1084. https://doi.org/10.1016/S01429612(00)00350-1

Bartolini, L., Iannuzzi, D., Mattei, G., 2018. Comparison of frequency and strain-rate domain mechanical characterization. Sci. Rep. 8, 13697. https://doi.org/10.1038/s41598-018-31737-3

Bhattacharya, S., Srivastava, A., Pal, A., 2006. Modulation of viscoelastic properties of physical gels by nanoparticle doping: Influence of the nanoparticle capping agent. Angew. Chemie - Int. Ed. 45, 29342937. https://doi.org/10.1002/anie.200504461

Cameron, A.R., Frith, J.E., Cooper-White, J.J., 2011. The influence of substrate creep on mesenchymal stem cell behaviour and phenotype. Biomaterials 32, 5979-5993. https://doi.org/10.1016/j.biomaterials.2011.04.003 
Campolo, J., Vozzi, F., Penco, S., Cozzi, L., Caruso, R., Domenici, C., Ahluwalia, A., Rial, M., Marraccini, P., Parodi, O., 2014. Vascular Injury Post Stent Implantation: Different Gene Expression Modulation in Human Umbilical Vein Endothelial Cells (HUVECs) Model. PLoS One 9, e90213. https://doi.org/10.1371/journal.pone.0090213

Charrier, E.E., Pogoda, K., Wells, R.G., Janmey, P.A., 2018. Control of cell morphology and differentiation by substrates with independently tunable elasticity and viscous dissipation. Nat. Commun. 9, 1-13. https://doi.org/10.1038/s41467-018-02906-9

Chaudhuri, O., Gu, L., Darnell, M., Klumpers, D., Sidi, A., Weaver, J.C., Huebsch, N., Mooney, D.J., Francisco, S., 2015. Substrate stress relaxation regulates cell spreading. Nat. Commun. 6. https://doi.org/10.1038/ncomms7365.Substrate

Dursun, S., Erdener, K., Nurettin, S., Olgun, G., 2004. The Influence of Preparation Methods on the Swelling and Network Properties of Acrylamide Hydrogels with Crosslinkers. J. Macromol. Sci. Part A Pure Appl. Chem. A41, 419-431. https://doi.org/10.1081/MA-120028476

Engler, A.J., Sen, S., Sweeney, H.L., Discher, D.E., 2006. Matrix Elasticity Directs Stem Cell Lineage Specification. Cell 126, 677-689. https://doi.org/10.1016/j.cell.2006.06.044

Engler, A.J., Sen, S., Sweeney, H.L., Discher, D.E., 2006. Matrix Elasticity Directs Stem Cell Lineage Specification. Cell 126.4, 677-689. https://doi.org/10.1016/j.cell.2006.06.044

Guo, C., Zhou, L., Lv, J., 2013. Effects of expandable graphite and modified ammonium polyphosphate on the flame-retardant and mechanical properties of wood flour-polypropylene composites. Polym. Polym. Compos. 21, 449-456. https://doi.org/10.1002/app

Hammond, B.F., 1969. Dextran production by a human oral strain of Lactobacillus casei. Arch. Oral Biol. 14, 879-890. https://doi.org/10.1016/0003-9969(69)90266-0

Hoffman, A.S., 2012. Hydrogels for biomedical applications. Adv. Drug Deliv. Rev. 64, 18-23. https://doi.org/10.1016/j.addr.2012.09.010

Humphrey, J.D., Dufresne, E.R., Schwartz, M.A., 2014. Mechanotransduction and extracellular matrix homeostasis. Nat. Rev. Mol. Cell Biol. 15, 802-812. https://doi.org/10.1038/nrm3896

Ingber, D.E., 1997. Tensegrity: the Architectural Basis of Cellular Mechanotransduction. Annu. Rev. Physiol. 59, 575-599. https://doi.org/10.1146/annurev.physiol.59.1.575

Jay, J.I., Shukair, S., Langheinrich, K., Hanson, M.C., Cianci, G.C., Johnson, T.J., Clark, M.R., Hope, T.J., Kiser, P.F., 2009. Modulation of Viscoelasticity and HIV Transport as a Function of $\mathrm{pH}$ in a Reversibly Crosslinked Hydrogel. Adv. Funct. Mater. 19, 2969-2977. https://doi.org/10.1002/adfm.200900757

Keplinger, C., Yun Sun, J., Chiang Foo, C., Josef Michael Rothemund, P., Whitesides, G.M., Keplinger, C., Sun, J.-Y., Suo, Z., 2013. Stretchable, Transparent, Ionic Conductors. Science (80-. ). 341, 984-987. https://doi.org/10.1126/science. 1240228

Kulicke, W.-M., Clasen, C., 2013. Viscosimetry of polymers and polyelectrolytes. Springer Science \& Business Media.

Kumar, S., 2014. Cellular mechanotransduction: Stiffness does matter. Nat. Mater. 13, 918-920. https://doi.org/10.1038/nmat4094

Liepsch, D., Thurston, G., Lee, M., 1991. Studies of fluids simulating blood-like rheological properties and applications in models of arterial branches. Biorheology 28, 39-52.

Mattei, G., Cacopardo, L., Ahluwalia, A., 2017. Micro-Mechanical Viscoelastic Properties of Crosslinked Hydrogels Using the Nano-Epsilon Dot Method. Materials (Basel). 10, 889. https://doi.org/10.3390/ma10080889

Naessens, M., Cerdobbel, A., Soetaert, W., Vandamme, E.J., 2005. Review Leuconostoc dextransucrase and dextran: production, properties and applications. J. Chem. Technol. Biotechnol. J Chem Technol 
Biotechnol 80, 845-860. https://doi.org/10.1002/jctb.1322

Nemir, S., West, J.L., 2009. Synthetic Materials in the Study of Cell Response to Substrate Rigidity. Ann. Biomed. Eng. 38, 2-20. https://doi.org/10.1007/s10439-009-9811-1

Nijenhuis, K. te, 1997. Agarose, in: Thermoreversible Networks: Viscoelastic Properties and Structure of Gels. Springer, Berlin, Heidelberg, pp. 194-202. https://doi.org/https://doi.org/10.1007/BFb0008710

Pek, Y.S., Wan, A.C.A., Ying, J.Y., 2010. The effect of matrix stiffness on mesenchymal stem cell differentiation in a 3D thixotropic gel. Biomaterials 31, 385-391. https://doi.org/10.1016/j.biomaterials.2009.09.057

Sabbahi, A., Vergnaud, J.M., 1993. Absorption of water by polyurethane foam. modelling and experiments. Eur. Polym. J. 29, 1243-1246. https://doi.org/10.1016/0014-3057(93)90155-9

Tirella, a., Mattei, G., Ahluwalia, A., 2013. Strain rate viscoelastic analysis of soft and highly hydrated biomaterials. J. Biomed. Mater. Res. - Part A 102A, 3352-3360. https://doi.org/10.1002/jbm.a.34914

Tse, J.R., Engler, A.J., 2010. Preparation of Hydrogel Substrates with Tunable Mechanical Properties. Curr. Protoc. Cell Biol. 1-16. https://doi.org/10.1002/0471143030.cb1016s47

Wells, R.G., 2008. The role of matrix stiffness in regulating cell behavior. Hepatology 47, 1394-1400. https://doi.org/10.1002/hep.22193

Wu, S., Shanks, R.A., 2003. Conformation of polyacrylamide in aqueous solution with interactive additives and cosolvents. J. Appl. Polym. Sci. 89, 3122-3129. https://doi.org/10.1002/app.12528

Yang, M.T., Fu, J., Wang, Y.-K., Desai, R.A., Chen, C.S., 2011. Assaying stem cell mechanobiology on microfabricated elastomeric substrates with geometrically modulated rigidity. Nat. Protoc. 6. https://doi.org/10.1038/nprot.2010.189

Zaman, M.H., Trapani, L.M., Sieminski, A.L., Mackellar, D., Gong, H., Kamm, R.D., Wells, A., Lauffenburger, D.A., Matsudaira, P., 2006. Migration of tumor cells in 3D matrices is governed by matrix stiffness along with cell-matrix adhesion and proteolysis. Proc. Natl. Acad. Sci. U. S. A. 103, 15-16. https://doi.org/Doi 10.1073/Pnas.0606087103

Zeltmann, S.E., Bharath Kumar, B.R., Doddamani, M., Gupta, N., 2016. Prediction of strain rate sensitivity of high density polyethylene using integral transform of dynamic mechanical analysis data. Polym. (United Kingdom) 101, 1-6. https://doi.org/10.1016/j.polymer.2016.08.053 


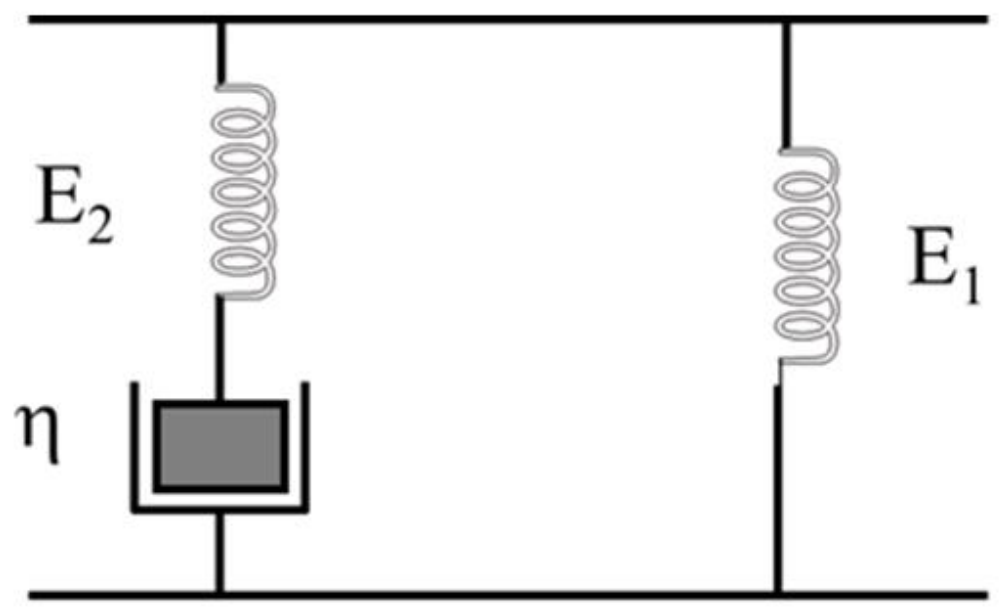

$$
\begin{gathered}
\mathbf{E}_{\text {inst }}=E_{1}+E_{2} \\
\mathbf{E}_{\text {eq }}=E_{1} \\
\tau=\frac{\eta}{E_{2}}
\end{gathered}
$$

Figure 1: The SLS model and symbols used to describe the lumped parameters obtained from fitting experimental data to the epsilon dot equations. Note that a lower relaxation time $\tau$ denotes a more viscous-like behaviour (i.e. when $\tau \rightarrow 0$ the material is ideally viscous and vice versa when $\tau \rightarrow \infty$ we have an ideally elastic material).

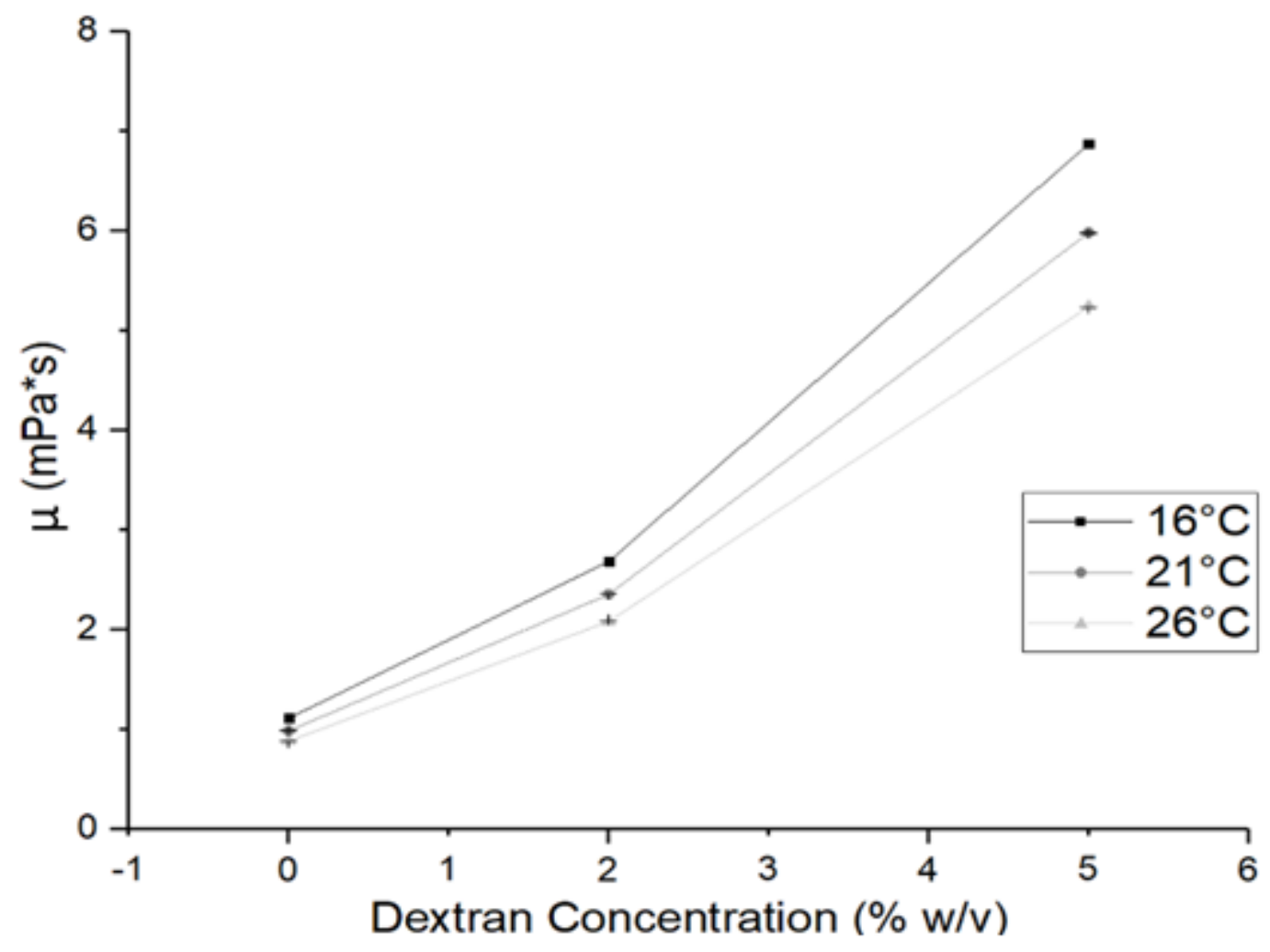

Figure 2: Dynamic viscosity of the dextran solutions as a function of concentration. 


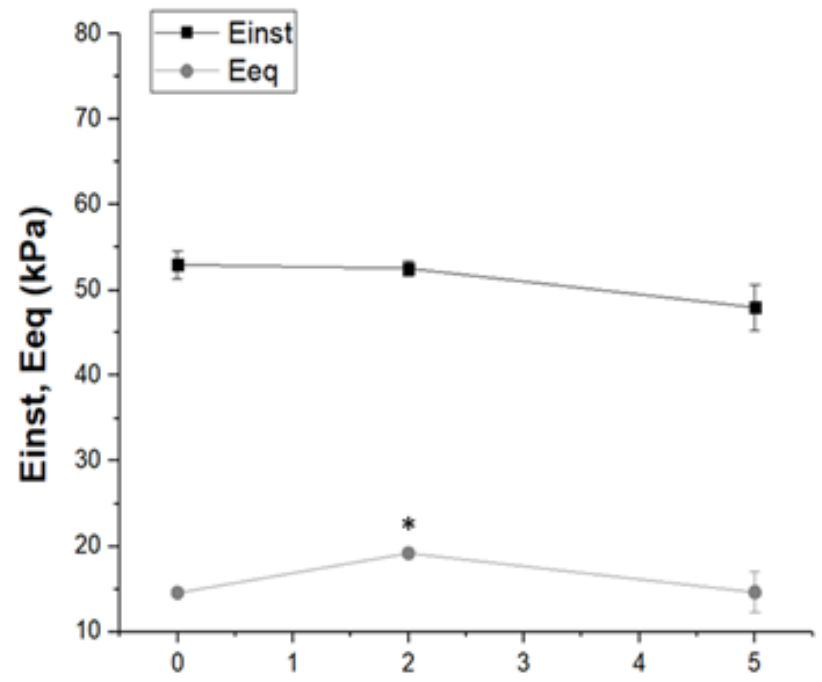

A Dextran Concentration (\% w/v)

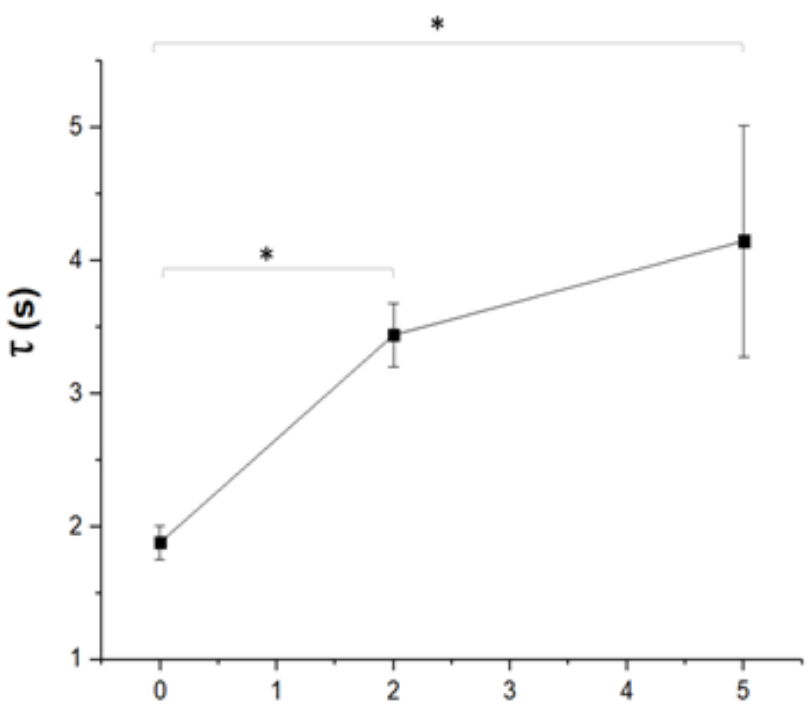

B Dextran Concentration $(\% \mathrm{w} / \mathrm{v})$

Figure 3: Instantaneous and equilibrium elastic moduli (A) and characteristic relaxation time (B) of sponges as a function of different dextran concentrations. A) $*=p<0.05$ with respect to other values of Eeq; B) $*=p<0.05$.

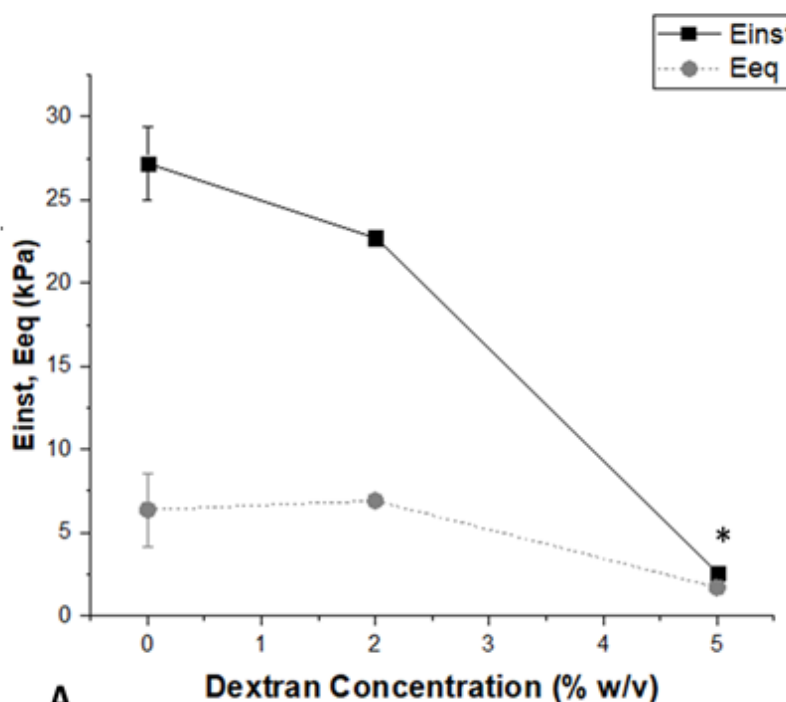

A

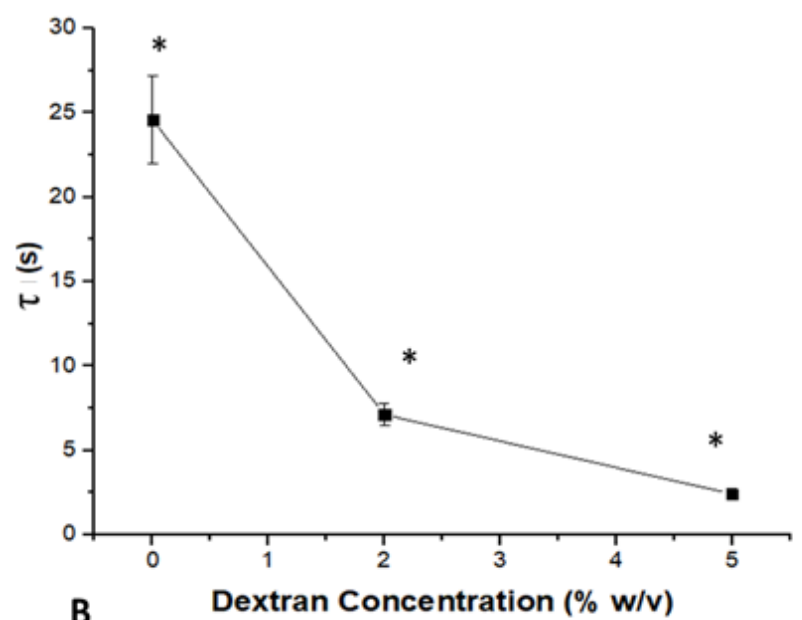

B

Figure 4: Instantaneous and equilibrium elastic moduli (A) and characteristic relaxation time (B) of Agarose samples as a function of different dextran concentrations. ${ }^{*}=\mathrm{p}<0.05$ with respect to other values of Einst, Eeq and $\tau$ respectively. 

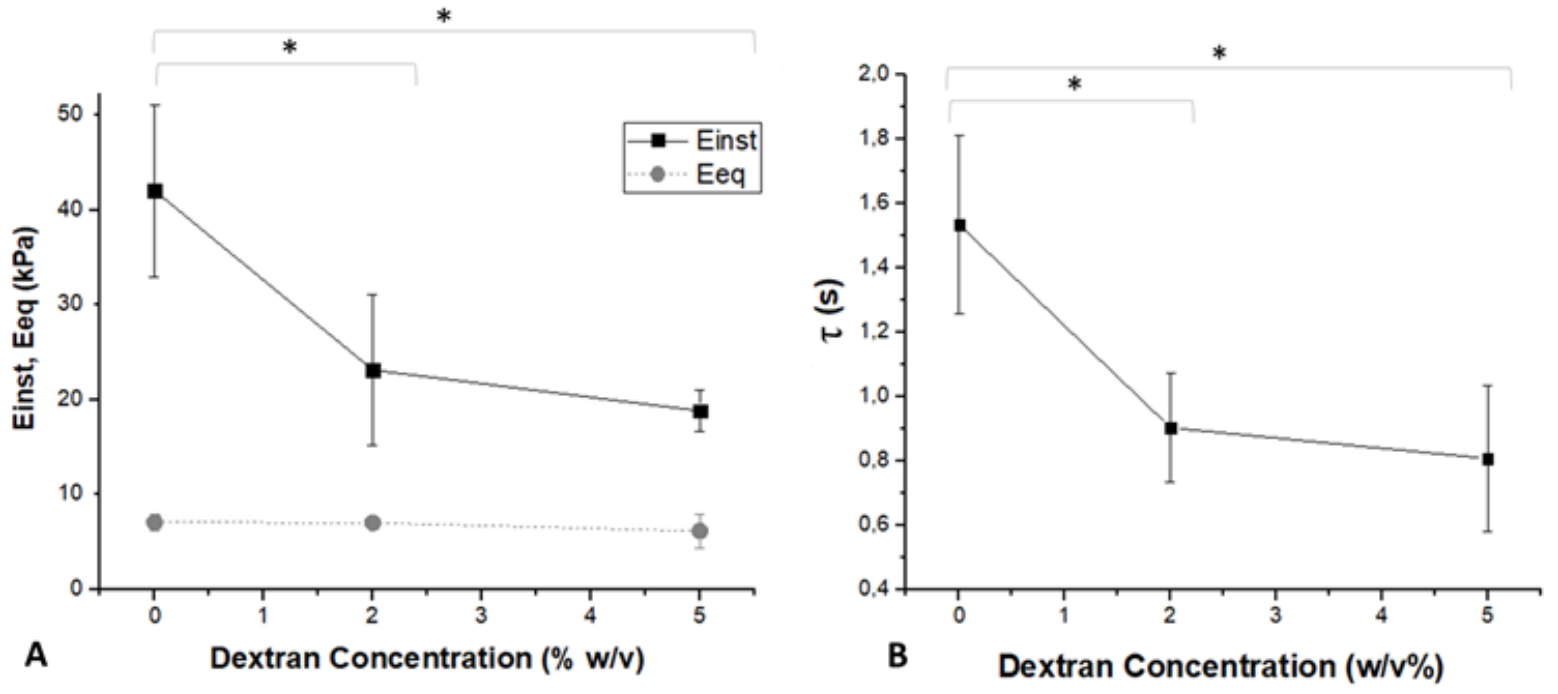

Figure 5: Instantaneous and equilibrium elastic moduli (A) and characteristic relaxation time (B) of PAAm samples as a function of different dextran concentrations. $*=p<0.05$.

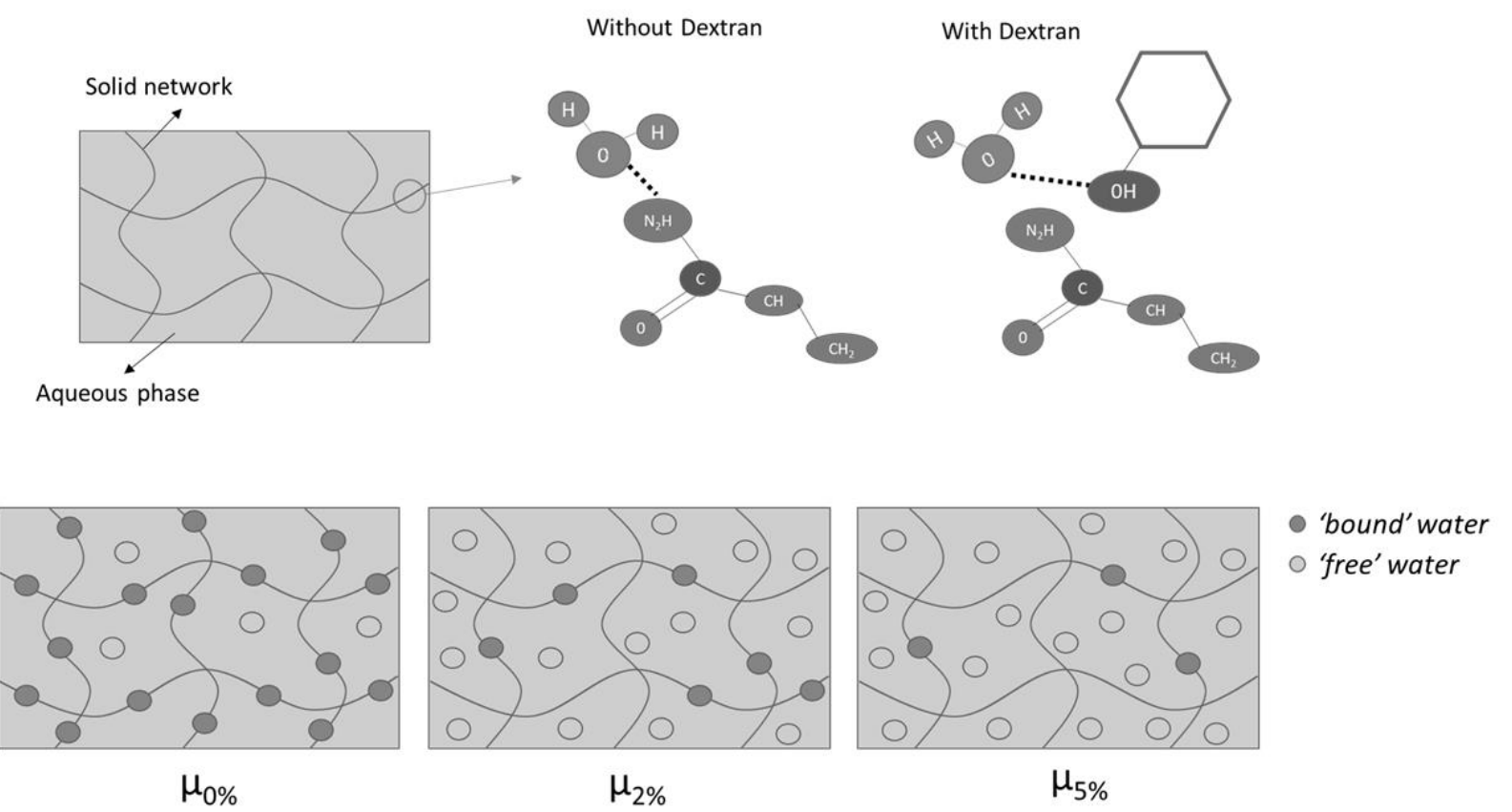

Figure 6: Schematic representation of the interaction between the solid network of PAAm and the liquid phase: without dextran hydrogen bonds are formed between acrylamide and water. In the presence of dextran, water tends to form the bonds with dextran rather than with acrylamide. Thus, the rate of movement and redistribution of the aqueous phase increases with increasing dextran concentration $(\mu 0 \%<\mu 2 \%<\mu 5 \%)$. Free water molecules are shown as empty circles, while bound water ones are represented as filled circles attached to the hydrogel polymer network (solid lines). 


\section{Graphical Abstract}
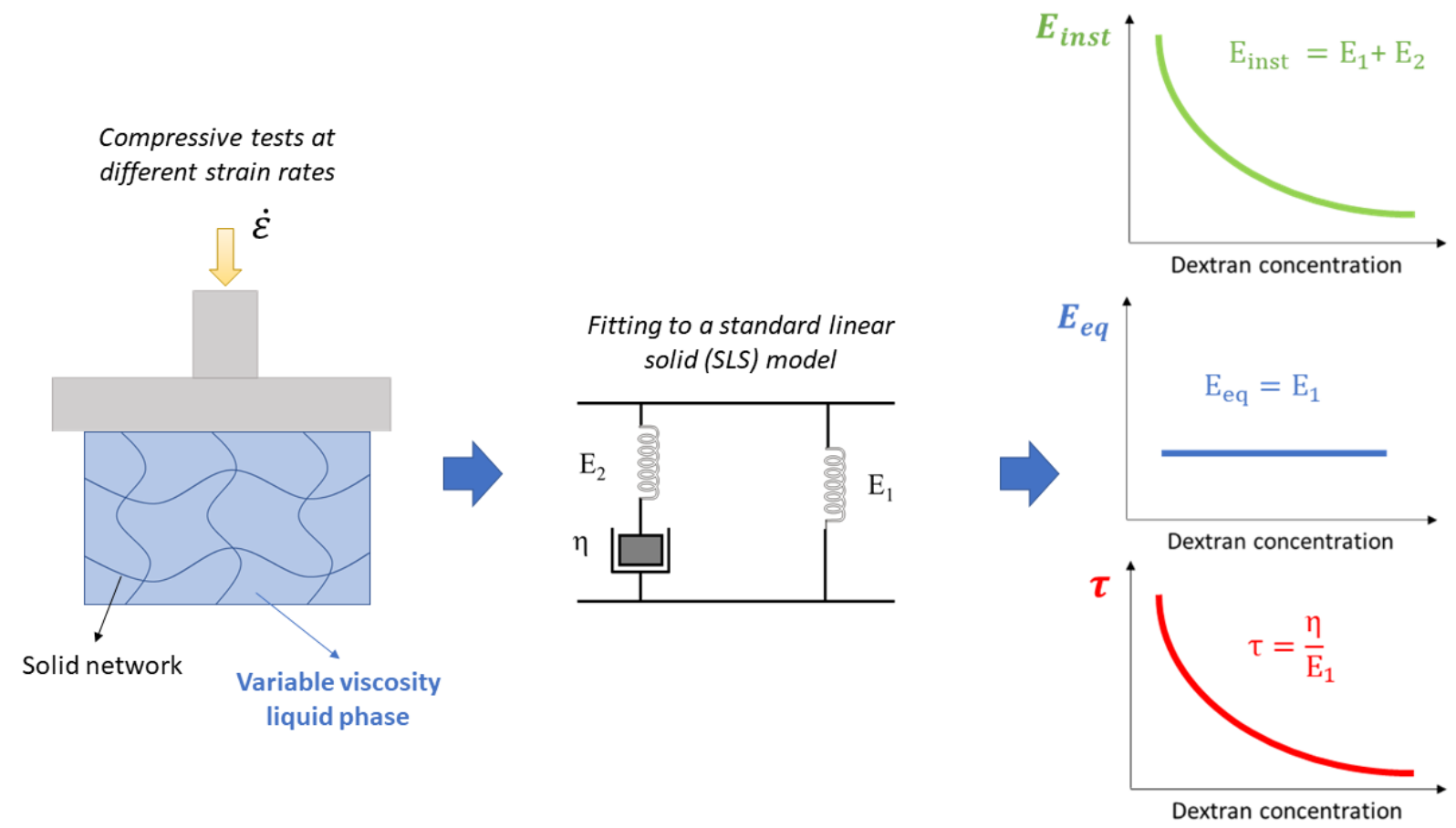